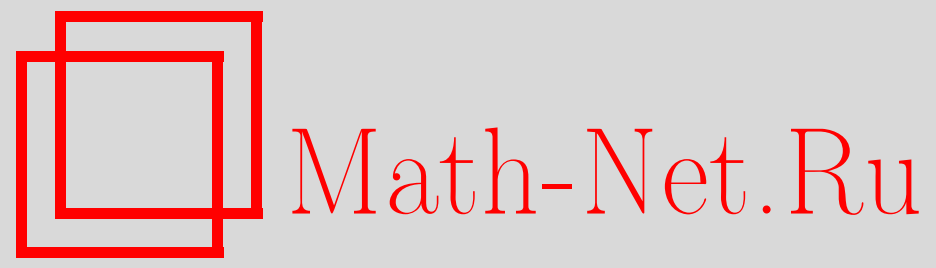

Обцероссийский математический портал 
Г. С. Чакветадзе, Стохастическая устойчивость инвариантной меры в одномерной модели бурения, Матем. заметки, 1998, том 64, выпуск 3, 477-480

DOI: https://doi.org/10.4213/mzm1422

Использование Общероссийского математического портала Math-Net.Ru подразумевает, что вы прочитали и согласны с пользовательским соглашением 
http://www. mathnet.ru/rus/agreement

Параметры загрузки:

IP: 3.80 .181 .102

26 апреля 2023 г., 14:17:05 


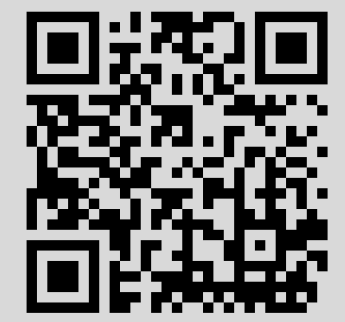




\section{СТОХАСТИЧЕСКАЯ УСТОЙЧИВОСТЬ ИНВАРИАНТНОЙ МЕРЫ В ОДНОМЕРНОЙ МОДЕЛИ БУРЕНИЯ}

\section{Г. Чакветадзе}

Исследуются стохастические возмущения одномерного отображения, моделирующего процесс бурения и введенного А. Лясотой и П. Русеком в [1]. Пусть $p: \mathbb{R} \mapsto \mathbb{R}-1$-периодическая, выпуклая и трижды непрерывно дифференцируемая на $[0,1]$ функция такая, что $p(1-r)=p(r), r \in \mathbb{R}$. Для $s \in \mathbb{R}$ через $\tau(s)$ обозначим абсциссу ближайшей справак $s$ точки пересечения графиков функций $p$ и $h_{s}$, где

$$
h_{s}(r)=p(s)+p^{\prime}(s+0)(r-s)-\frac{\lambda}{2}(r-s)^{2}, \quad \lambda<\inf _{r \in[0,1]}\left|p^{\prime \prime}(r)\right| .
$$

Положим $W(s)=\tau(s) \bmod 1, s \in[0,1)$. Ниже будем предполагать, что параметр $\lambda$ удовлетворяет условию (В) теоремы в [2]. В этом случае график $W$ описывается следующей леммой. 
Лемма 1. Существуют точки $s_{0}, \ldots, s_{n}, 0<s_{n}<\cdots<s_{1}<1 / 2<s_{0}<1, n \geqslant 2, u$ $\varepsilon_{0}>0$ maкue, чmo

1) $W$ непрерывно дифференцируемо и строго убъвает на интервалах $I_{k}=\left(s_{k}, s_{k-1}\right)$, $k=1, \ldots, n$;

2) $\left(W(s)-\varepsilon_{0}, W(s)+\varepsilon_{0}\right) \subset I$ для всех $s \in I=\left[s_{n}, s_{0}\right]$;

3) $\sup _{s \in I}\left(\left|W^{\prime}(s)\right|\right)^{-1}<\infty u \operatorname{dist}\left(W^{l}(s), I\right) \rightarrow 0$ npu $l \rightarrow \infty$ длs всеx $s \in(0,1)$.

Пусть $w=\left.W\right|_{I}, L_{1}=L_{1}(I, d s), L_{\infty}=L_{\infty}(I, d s)$,

$$
B V=\left\{f \in L_{1}: v(f)=\inf _{\widehat{f} \in f} \operatorname{Var} \widehat{f}<\infty\right\} .
$$

Через $\|\cdot\|_{\gamma}=\gamma v(f)+|\cdot|_{1}\left(|\cdot|_{1}-\right.$ норма в $\left.L_{1}\right), \gamma \in(0,1]$, обозначим норму, которая превращает $B V$ в банахово пространство. Для линейного ограниченного оператора $T:\left(B V,\|\cdot\|_{1}\right) \rightarrow(B V$, $\left.\|\cdot\|_{1}\right)$ через $\sigma(T)$ обозначается спектр $T$. Оператор Перрона-Фробениуса $P: L_{1} \rightarrow L_{1}$ определяется равенством

$$
\int g \cdot P f d s=\int g \circ w \cdot f d s, \quad f \in L_{1}, \quad g \in L_{\infty} .
$$

В принятых предположениях о значениях $\lambda 1$ есть простое собственное значение $P: L_{1} \rightarrow L_{1}$ с собственной функцией $\varrho \in B V$, являющейся плотностью конечной абсолютно непрерывной инвариантной меры $\mu$ отображения $w$. Кроме того, $P(B V) \subset B V$, оператор $P$ ограничен в $\left(B V,\|\cdot\|_{1}\right)$ и $\varkappa=\sup \{|z|: z \in \sigma(P) \backslash\{1\}\}<1$.

Одним из критериев адекватности введенной динамической модели бурения служит устойчивость меры $\mu$ относительно семейства случайных возмущений $\Upsilon_{\vec{\varepsilon}}, \vec{\varepsilon}=\left(\varepsilon_{x}, \varepsilon_{y}\right)$, определяемого следуюшим образом. Предположим, что заданы малые отрезки $J_{x}, J_{y}$ и функции $v_{1}: I \times J_{x} \times J_{y} \rightarrow \mathbb{R}$, $v_{2}: I \times J_{y} \rightarrow \mathbb{R}$ такие, что

a) $v_{i}, \partial v_{i} / \partial s, \partial^{2} v_{i} / \partial s^{2}$ непрерьвно зависят от $s, x, y$;

б) для всех $s \in I, y \in J_{y}$ функция $v_{1}(s, \cdot, y)$ непрерывно дифференцируема и строго возрастает на $J_{x}$

в) $v_{1}(s, 0,0)=p(s), v_{2}(s, 0)=p^{\prime}(s)$.

Зафиксируем $s \in I$ и введем борелевские функции $\tau(s, \cdot, \cdot): J_{x} \times J_{y} \rightarrow \mathbb{R}, w(s, \cdot, \cdot): J_{x} \times J_{y} \rightarrow \mathbb{R}$. А именно, для любой пары $\langle x, y\rangle \in J_{x} \times J_{y}$ через $\tau(s, x, y)$ обозначим абсциссу ближайшей справа к 1 точки пересечения основного профиля и графика функции

$$
h_{s, x, y}(r)=v_{1}(s, x, y)+v_{2}(s, y)(r-s)-\frac{\lambda}{2}(r-s)^{2} ;
$$

положим $w(s, x, y)=\tau(s, x, y) \bmod 1,\langle x, y\rangle \in J_{x} \times J_{y}$ (промежутки $J_{x}$ и $J_{y}$ предполагаются настолько малыми, что верно включение $\left.w\left(I \times J_{x} \times J_{y}\right) \subset I\right)$. Рассмотрим семейство случайных величин $w\left(s, \xi_{\vec{\varepsilon}}, \eta_{\vec{\varepsilon}}\right)$, где для любого $s \in I\left\langle\xi_{\vec{\varepsilon}}, \eta_{\vec{\varepsilon}}\right\rangle$ распределено на множестве $X_{\vec{\varepsilon}} \times Y_{\vec{\varepsilon}} \subset J_{x} \times J_{y}$, $X_{\vec{\varepsilon}}=\left[-\varepsilon_{x}, \varepsilon_{x}\right], Y_{\vec{\varepsilon}}=\left[-\varepsilon_{y}, \varepsilon_{y}\right]$, с плотностью $u_{\vec{\varepsilon}}(s, \cdot, \cdot)$, липшицевой по $s$ сконстантой $K=K\left(\varepsilon_{x}\right.$, $\left.\varepsilon_{y}\right)=o\left(1 /\left(\varepsilon_{x} \varepsilon_{y}\right)\right),|\vec{\varepsilon}|=\max \left(\varepsilon_{x}, \varepsilon_{y}\right) \rightarrow 0$. Для любого $\vec{\varepsilon}$ плотности $p_{\vec{\varepsilon}}(s, \cdot)$ распределения величин $w\left(s, \xi_{\vec{\varepsilon}}, \eta_{\vec{\varepsilon}}\right)$ могут быть взяты в качестве переходных плотностей вероятностей однородной марковской цепи $\Upsilon_{\vec{\varepsilon}}$. Для исследования асимптотики стационарных распределений $\varrho_{\vec{\varepsilon}}$ цепей $\Upsilon_{\vec{\varepsilon}}$ при $|\vec{\varepsilon}| \rightarrow 0$ введем марковские операторы $P_{\vec{\varepsilon}}: L_{1} \rightarrow L_{1}$ по формуле

$$
P_{\vec{\varepsilon}} f(r)=\int f(s) p_{\vec{\varepsilon}}(s, r) d s .
$$

ТЕорема. Предположим, что отображсение $w$ не имеет периодических точек разрыва. Тогда для достаточно малого $|\vec{\varepsilon}| 1$ есть простое собственное значение оператора $P_{\vec{\varepsilon}}: L_{1} \rightarrow L_{1}$ с собственной функиией $\varrho_{\vec{\varepsilon}} \in B V$, являющейся плотностью стационарного распределения цепи $\Upsilon_{\vec{\varepsilon}} ; P_{\vec{\varepsilon}}$ ограничены в $B V, \varkappa_{\vec{\varepsilon}}=\sup \left\{|z|: z \in \sigma\left(P_{\vec{\varepsilon}}\right) \backslash\{1\}\right\}<1$. Кроме того,

1) $\left|\varrho_{\vec{\varepsilon}}-\varrho\right|_{1} \rightarrow 0,|\vec{\varepsilon}| \rightarrow 0$

2) $\varlimsup_{|\vec{\varepsilon}| \rightarrow 0} \varkappa_{\vec{\varepsilon}}<\varkappa^{1 / 2}$. 
Доказательство теоремы содержится в леммах 2-4. Предварим формулировку первой из них следующим замечанием. При достаточно малых $x, y$ функция $w(\cdot, x, y)$ задает кусочно-монотонное преобразование $w_{x, y}$ промежутка $I$ в себя. Семейства $\left\{s_{k, x, y}\right\}_{k=0}^{n}$ и $\left\{I_{k, x, y}\right\}_{k=1}^{n}$ точек разрыва и интервалов монотонности отображений $w_{x, y}$ находятся в биективном соответствии с семействами $\left\{s_{k}\right\}_{k=0}^{n}$ и $\left\{I_{k}\right\}_{k=1}^{n}$, причем для любого $k$ множества $\left\{(s, r): s \in I_{k, x, y}, r=w_{x, y}(s)\right\}$ сходятся в хаусдорфовой метрике к множествам $\left\{(s, r): s \in I_{k}, r=w(s)\right\}$. Положим $\alpha=\langle x, y\rangle$, $|\alpha|=\max (|x|,|y|), w_{k, \alpha}=\left.w_{\alpha}\right|_{I_{k, \alpha}}, g=\left|w^{\prime}\right|^{-1}$,

$$
g_{\alpha}(s)=\left|\frac{\partial}{\partial s} w(s, x, y)\right|^{-1}, \quad u_{\vec{\varepsilon}, \alpha}=u_{\vec{\varepsilon}}(\cdot, x, y), \quad \int \cdot d \alpha=\int_{X_{\varepsilon}} \int_{Y_{\varepsilon}} \cdot d x d y .
$$

ЛЕмма 2. Для $k \in\{1, \ldots, n\}$

1) при достаточно мальх $|\alpha|$ функщия $g_{\alpha} \circ w_{k, \alpha}^{-1}$ липшицева на промежутке $w_{\alpha}\left(I_{k, \alpha}\right)$ с константой $C_{1}$, не зависящей от $\alpha$;

2) для любого $\delta>0$ найдется $\varepsilon_{1}=\varepsilon_{1}(\delta)$ такое, что если $|\alpha|<\varepsilon_{1}, s \in I_{k}, s^{\prime} \in I_{k, \alpha}$, $\left|s-s^{\prime}\right|<\varepsilon_{1}$, mo $\left|g(s)-g_{\alpha}\left(s^{\prime}\right)\right|<\delta$.

Следующая лемма дает удобную формулу для вычисления $P_{\vec{\varepsilon}}^{l}$. Для набора $\vec{\alpha}=\left(\alpha_{1}, \ldots, \alpha_{l}\right)=$ $\left(\left\langle x_{1}, y_{1}\right\rangle, \ldots,\left\langle x_{l}, y_{l}\right\rangle\right)$ положим

$$
\begin{gathered}
w_{\vec{\alpha}}^{l}=w_{\alpha_{l}} \circ \cdots \circ w_{\alpha_{1}}, \quad g_{\vec{\alpha}}^{l}(s)=g_{\alpha_{1}}(s) \cdots g_{\alpha_{l}}\left(w_{\left(\alpha_{1}, \ldots, \alpha_{l-1}\right)}^{l-1}(s)\right), \\
u_{\vec{\varepsilon}, \vec{\alpha}}^{l}(s)=u_{\vec{\varepsilon}, \alpha_{1}}(s) \cdots u_{\vec{\varepsilon}, \alpha_{l}}\left(w_{\left(\alpha_{1}, \ldots, \alpha_{l-1}\right)}^{l-1}(s)\right) .
\end{gathered}
$$

Лемма 3. Справедливо равенство

$$
P_{\vec{\varepsilon}}^{l} f(r)=\int \cdots \int d \alpha_{1} \cdots d \alpha_{l} P_{\vec{\alpha}}^{l} f(r)
$$

əəe

$$
P_{\vec{\alpha}}^{l} f(r)=\sum_{s: w_{\vec{\alpha}}^{l}(s)=r} f(s) g_{\vec{\alpha}}^{l}(s) u_{\vec{\varepsilon}, \vec{\alpha}}^{l}(s) .
$$

Следующая лемма позволяет применить к нашей ситуации леммы теории возмущений из [3]. Положим (см. [4])

$$
\theta=\lim _{l \rightarrow \infty}\left(\sup _{s \in I} g(s) \cdots g\left(w^{l-1}(s)\right)\right)^{1 / l}<1 .
$$

Лемма 4. Пусть $\theta<\Lambda$. Тогда существуют $C>0, L \in \mathbb{N}$ такие, что для всех $l \geqslant L$ найдется $\varepsilon_{2}=\varepsilon_{2}(l)$ maкое, что $\left\|P_{\vec{\varepsilon}}^{l}-P^{l}\right\|_{\Lambda^{l}} \leqslant C \Lambda^{l},|\vec{\varepsilon}|<\varepsilon_{2}$.

ДокАЗАТЕЛЬСтво. Обозначим через $c_{i}=c_{i}(\vec{\varepsilon})$ функцию, бесконечно малую при $|\vec{\varepsilon}| \rightarrow 0$. Зафииксируем $\Theta, \theta<\Theta<\Lambda$. Покажем, что существуют $L_{1}, L_{2} \in \mathbb{N}$ и $D_{1}, D_{2}>0$ такие, что

$$
\Lambda^{l} \operatorname{Var} P^{l} f<\left(\Theta^{l}+\Lambda^{l} D_{1}\right)\left(\Lambda^{l} \operatorname{Var} f+|f|_{1}\right), \quad l \geqslant L_{1},
$$

и для всех $l \geqslant L_{2}$ найдется $\varepsilon_{3}=\varepsilon_{3}(l)$ такое, что

$$
\begin{gathered}
\Lambda^{l} \operatorname{Var} P_{\vec{\varepsilon}}^{l} f<\left(\Theta^{l}+\Lambda^{l} D_{2}\right)\left(\Lambda^{l} \operatorname{Var} f+|f|_{1}\right), \quad|\vec{\varepsilon}|<\varepsilon_{3}, \\
\left|P_{\vec{\varepsilon}}^{l} f-P^{l} f\right|_{1} \leqslant c_{1}\|f\|_{\Lambda^{l}} .
\end{gathered}
$$

Оценка (1) вытекает из предложения 1 в [5]. Поясним вывод (2). Используя леммы 3 и 4 из [5], получим

$$
\begin{aligned}
\Lambda^{l} \operatorname{Var} P_{\vec{\varepsilon}}^{l} f & \leqslant \Lambda^{l} \int \cdots \int d \alpha_{1} \cdots d \alpha_{l} \operatorname{Var} P_{\vec{\alpha}}^{l} f \leqslant \Lambda^{l} \int \cdots \int d \alpha_{1} \cdots d \alpha_{l} \operatorname{Var} f g_{\vec{\alpha}}^{l} u_{\vec{\varepsilon}, \vec{\alpha}}^{l} \\
& \leqslant \Lambda^{l} \int \cdots \int d \alpha_{1} \cdots d \alpha_{l}\left(\Delta_{\vec{\varepsilon}, \vec{\alpha}}^{l} \operatorname{Var} f g_{\vec{\alpha}}^{l}+\Omega_{\vec{\varepsilon}, \vec{\alpha}}^{l} \sup f g_{\vec{\alpha}}^{l}\right),
\end{aligned}
$$


где

$$
\begin{gathered}
\Delta_{\vec{\varepsilon}, \vec{\alpha}}^{l}=\sup u_{\vec{\varepsilon}, \vec{\alpha}}^{l} \leqslant \prod_{i=1}^{l} \sup u_{\vec{\varepsilon}}\left(\cdot, x_{i}, y_{i}\right) \\
\Omega_{\vec{\varepsilon}, \vec{\alpha}}^{l}=\sum_{J \in S_{\vec{\alpha}}^{l}} \operatorname{Var}_{J} u_{\vec{\varepsilon}, \vec{\alpha}}^{l} \leqslant \sum_{j=1}^{l} \operatorname{Var} u_{\vec{\varepsilon}}\left(\cdot, x_{j}, y_{j}\right) \prod_{i \neq j} \sup u_{\vec{\varepsilon}}\left(\cdot, x_{i}, y_{i}\right) \cdot N_{\vec{\alpha}}^{l},
\end{gathered}
$$

$S_{\vec{\alpha}}^{l}$ - семейство интервалов монотонности композиции $w_{\vec{\alpha}}^{l}, N_{\vec{\alpha}}^{l}=\operatorname{card} S_{\vec{\alpha}}^{l}$. Используя лемму 2 и рассуждая так же, как и при вьводе оценки (5.8) в [3], найдем $L_{2} \in \mathbb{N}, D_{2}>0, \delta>0$ такие, что при $l \geqslant L_{2}$ и малом $\varepsilon$

$$
\operatorname{Var} f g_{\vec{\alpha}}^{l}<(\Theta-\delta)^{l} \operatorname{Var} f+\left(D_{2}-\delta\right)|f|_{1}, \quad \max _{1 \leqslant i \leqslant l}\left|\alpha_{i}\right|<\varepsilon
$$

Так как

$$
\begin{aligned}
& N_{\vec{\alpha}}^{l} \leqslant n^{l}, \quad \iint d x d y u_{\vec{\varepsilon}}\left(\frac{1}{2}, x, y\right)=1, \\
\iint d x d y \sup u_{\vec{\varepsilon}}(\cdot, x, y)= & \iint d x d y u_{\vec{\varepsilon}}\left(\frac{1}{2}, x, y\right)+\iint d x d y\left(\sup u_{\vec{\varepsilon}}(\cdot, x, y)-u_{\vec{\varepsilon}}\left(\frac{1}{2}, x, y\right)\right) \\
\leqslant & 1+\frac{K}{2} \cdot 4 \varepsilon_{x} \varepsilon_{y},
\end{aligned}
$$

то

$$
\begin{gathered}
\int \cdots \int d \alpha_{1} \cdots d \alpha_{l} \Delta_{\vec{\varepsilon}, \vec{\alpha}}^{l} \leqslant\left(1+2 K \varepsilon_{x} \varepsilon_{y}\right)^{l} \rightarrow 1, \\
\int \cdots \int d \alpha_{1} \cdots d \alpha_{l} \Omega_{\vec{\varepsilon}, \vec{\alpha}}^{l} \leqslant\left(1+2 K \varepsilon_{x} \varepsilon_{y}\right)^{l-1} 4 K \varepsilon_{x} \varepsilon_{y} l n^{l} \rightarrow 0,
\end{gathered}
$$

Соединяя (4) и (5), получаем (2).

С помощью лемм 2 и 7 из [6] находим, что

$$
\left|P_{\vec{\varepsilon}} f-P f\right|_{1} \leqslant c_{2}\|f\|_{1} .
$$

При $l \geqslant 2$ имеем $\left|P_{\vec{\varepsilon}}^{l} f-P^{l} f\right|_{1} \leqslant\left|P_{\vec{\varepsilon}}^{l-1}\left(P_{\vec{\varepsilon}}-P\right) f\right|_{1}+\left|\left(P_{\vec{\varepsilon}}^{l-1}-P^{l-1}\right) P f\right|_{1}$. Так как $\left|P_{\vec{\varepsilon}}\right|_{1}=1$ и $\|P f\|_{1} \leqslant$ const $\|f\|_{1}$, действуя по индукции, получаем неравенство $\left|P_{\vec{\varepsilon}}^{l} f-P^{l} f\right|_{1} \leqslant c_{3}\|f\|_{1}$, после чего оценка (3) вытекает из эквивалентности норм $\|\cdot\|_{\gamma}, 0<\gamma \leqslant 1$.

Рассуждая, как в доказательстве леммы 7 из [7], а также используя неравенства (1)-(3) и $\Theta<\Lambda$, получаем требуемую в лемме оценку с $L=\max \left(L_{1}, L_{2}\right), C=D_{1}+D_{2}+2$.

Утверждение теоремы вытекает из неравенства (6), леммы 4 и результатов [3, п. 5.Е] (см. также [8]).

Автор выражает глубокую признательность проф. А. М. Стёпину за постановку задачи, внимание к этой работе и полезные обсуждения.

\section{СПИСОК ЦИТИРОВАННОЙ ЛИТЕРАТУРЫ}

1. Lasota A., Rusek P. // Arch. Gornicktwa. 1974. V. 19. №3. P. 281-295. 2. Chakvetadze G. // J. Dynam. Control Systems. 1996. V. 2. № 4. P. 485-502. 3. Baladi V., Young L.-S. // Comm. Math. Phys. 1993. V. 156. P. 355-385. 4. Чакветадзе Г. // УМН. 1997. Т. 52. № 4. C. 223-224. 5. Rychlik M. // Studia Math. 1983. V. 76. P. 69-80. 6. Бланк М. Л. // Тр. семин. им. И. Г. Петровского. 1986. Т. 11. С. 166-189. 7. Hofbauer F., Keller G. // Math. Z. 1982. V. 180. P. 119-140. 8. Baladi V., Young L.-S. // Comm. Math. Phys. 1994. V. 166. P. 219-220. 